\title{
Bowel preparation with 1L polyethylene glycol and ascorbate NER1006 doubles the chance to detect three or more adenomas in overweight or obese males
}

\section{(ㄷ)(1) $(-)$}

Authors

Michael Epstein', Juha Halonen², Prateek Sharma ${ }^{3,4}$

Institutions

1 Investigative Clinical Research, Annapolis, Maryland, United States

2 Medical Affairs, Norgine Ltd., Harefield, United Kingdom

3 Department of Gastroenterology and Hepatology, Veterans Affairs Medical Center, Kansas City, Missouri, United States

4 Department of Gastroenterology and Hepatology, University of Kansas Medical Center, Kansas City, Kansas, United States

submitted 18.12 .2020

accepted after revision 12.4.2021

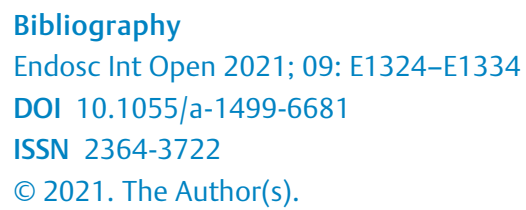

\section{ABSTRACT}

Background and study aims Men have more colon cleansing failures, colorectal adenomas, and colorectal cancers than women. We analyzed whether 1 -liter $(1 \mathrm{~L})$ polyethylene glycol (PEG) NER1006 improves high-quality (HQ) colon cleansing and adenoma detection in males versus two mid-volume alternatives.

Patients and methods The analysis of 1028 adult patients in two randomized clinical trials was performed. Adenoma detection and $\mathrm{HQ}$ cleansing were compared for overnight split dosing regimens with NER1006 $(n=513)$ versus combined oral sulfate solution or $2 \mathrm{~L}$ PEG + ascorbate (OSS/2 L PEG) $(n=515)$. Analyses included males versus females, overweight or obese $(\mathrm{OO})$ males versus lean males, and NER1006 versus OSS/2 L PEG. In male patients, the adenoma detection rate of at least $3($ ADR $3+)$ was predicted with multiple logistic regression and statistical comparisons used the two-sided t-test.

Results ADR3 + was greater in males versus females (10.7\% [56/524] versus $5.8 \%$ [29/504]; $\mathrm{P}=0.004)$ despite comparable adequate cleansing success rates (93.2\% [479/514] versus $93.0 \%$ [466/501]; $\mathrm{P}=0.912$ ) and more HQ-scores in females (41.6\% [1069/2570] versus 45.3\% [1134/2505]; P $=0.008)$. ADR was almost twice as high in OO versus lean males (43.4\% [184/424] versus $23.1 \%$ [21/91]; $\mathrm{P}<0.001)$. Multivariate logistic regression predicted ADR3 + detection to be twice as likely in $\mathrm{OO}$ males using NER 1006 versus OSS/ 2 L PEG (odds ratio (95\% confidence interval $[\mathrm{Cl}]$ ) $=2.049$ (1.082-3.973); $\mathrm{P}=0.030)$ and $90 \%$ more likely in all males (1.902 (1.045-3.526); $\mathrm{P}=0.037)$. In males, including $\mathrm{OO}$ males, NER1006 attained more HQ-scores per trial than OSS or $2 \mathrm{~L}$ PEG ( $\mathrm{P} \leq 0.017$ for all comparisons).

Conclusions NER1006 predicted the detection of more males for frequent surveillance than OSS/2 L PEG.

\section{Introduction}

Colorectal adenomas are associated with an increased risk for future advanced neoplasia and subsequent colorectal cancer.
An increased adenoma detection by colonoscopy can help reduce the risk of colorectal cancer [1, 2].

The smallest so-called diminutive adenomas $<5 \mathrm{~mm}$ in size are less likely to develop into malignancies. Recent evidence confirms the increased risk for advanced lesions, particularly 
when at least one adenoma out of a minimum of three is sized at least 6 to $9 \mathrm{~mm}[3,4]$. Recent clinical guidelines in the United States and Europe, therefore, suggest shorter surveillance intervals for these patients. The US Multi-Society Task Force, for post-colonoscopy follow-up in average-risk adults with normal colonoscopy or adenomas, now recommends shortening the standard 10-year screening/surveillance interval for patients without adenomas and for patients with three to four adenomas $<10 \mathrm{~mm}$ to 3 to 5 years and for patients with five such adenomas to only 3 years [5]. The European Society of Gastrointestinal Endoscopy (ESGE) now recommends no surveillance (e.g., return to screening every 10 years) for all patients with less than five adenomas [6].

Diminutive adenomas may be present but not always detected in bowels where cleansing is classified as only adequate on the Boston Bowel Preparation Scale (BBPS) and the Harefield Cleansing Scale (HCS), as stool-free high-quality (HQ) cleansing has been shown to improve both the adenoma detection rate and the mean number of adenomas detected per patient [7].

Patients aged 60 and higher have more adenomas than younger patients, although the younger age group under 50 years of age has recently shown a rapidly growing rate of colorectal cancer [8-11]. When looking at the incidence rates for colorectal cancer, the risk for colorectal cancer is higher in men compared with women and increases with a body mass in$\operatorname{dex}(\mathrm{BMI}) \geq 25[12,13]$.

With an estimated use of 1-liter polyethylene glycol (1LPEG) NER1006 (PLENVU ${ }^{\circledR}$ ) in over 3.5 million patients worldwide [14], NER1006 has been shown to provide superior HQ (e.g., stool-free score before any suction) colon cleansing efficacy versus combined oral sulfate solution or $2 \mathrm{~L}$ PEG + ascorbate (OSS/2 L PEG) and an improved cleansing quality versus 4L PEG [15-17].

\section{Patients and methods}

The further analysis of two highly similarly-designed phase 3 randomized clinical trials, MORA and NOCT, investigated the clinical benefits of bowel preparation with overnight split dosing from combined NER1006 arms versus combined 2 L PEG or OSS, mostly beyond the attained cleansing benefits that have been reported before [15-19]. MORA and NOCT are near-identical study designs, and the variance of the observed effects are likely to be similar across the trials.

We studied the comparative detection rates of colorectal polyps or adenomas in the overall colon and the right colon [16]. The right colon cleansing quality was an alternative primary endpoint in these trials. We focused our attention on two common adult patient groups: all males and overweight/ obese (OO) males.

We analyzed the data from both the MORA and NOCT trials on colonic polyp and adenoma counts assessed by the site endoscopists per their usual clinical practice. A total of 1028 patients with a documented polyp and adenoma counts in the overall colon and separately in the right colon were included in this analysis.

\section{Assessments}

The focus of this exploratory study was to identify the number of patients with three or more adenomas (adenoma detection rate $[A D R] 3+$ ) and separately five or more adenomas (ADR5 +) who may require shorter surveillance intervals $[5,6]$. We included several additional lesion detection analyses. The lesion detection rates (having at least one polyp or adenoma either overall (PDR and ADR, respectively) or in the right colon (rPDR and $\mathrm{rADR}$, respectively) were determined. All detection rates are reported as a percentage with the sum of positives (one per patient) divided by the analyzed sample size.

The mean overall number of polyps or adenomas per patient (MPP and MAP, respectively) and separately the mean number of polyps or adenomas in the right colon (rMPP and rMAP, respectively) were analyzed. The MPP and MAP were also analyzed in only polyp-positive (MPP + and rMPP +) or adenoma-positive $(M A P+$ and $r M A P+)$ patients. The results from all MPP, $\mathrm{MAP}, \mathrm{MPP}+, \mathrm{MAP}+$, rMPP, rMAP, rMAP+, and rMPP+ analyses are reported as means with $95 \%$ confidence intervals $(\mathrm{CI})$.

The attainment of overall adequate or HQ cleansing was assessed by comparing the percentage of patients who attained Grade $A B$ or $A$, respectively, on the validated HCS [20]. As a more detailed measure, segmental cleansing success was assessed as the number of HQ cleansed colon segments per treatment group, given that adenomas are more likely to be detected in HQ than adequate-quality cleansed colon segments [7]. We included in our analyses the cleansing scores rated by site colonoscopists and central readers.

\section{Lesion detection and cleansing outcomes in males versus females}

Among the 1028 patients with documented polyp and adenoma counts, 524 were males, and 504 were females.

The first analysis compared the polyp and adenoma detection parameters in males versus females. The attainment of overall adequate-quality or HQ cleansing was then assessed by comparing the percentage of patients who attained HCS Grade $A B$ or $A$, respectively. The second cleansing outcome was the number of HQ cleansed colon segments per treatment group.

\section{Lesion detection and cleansing outcomes in 00 versus lean males}

BMI data were available for 515 males. Patients were classified as $\mathrm{OO}$ if they had a BMI $\geq 25(n=424)$ and as lean if they had a $\mathrm{BMI}<25(\mathrm{n}=91)$. Lesion detection and cleansing quality data were analyzed and presented.

\section{Lesion detection and cleansing outcomes with NER 1006 vs OSS/2 L PEG}

The detailed analysis examined lesion detection with NER1006 versus OSS/2 L PEG - first in all males and then in those males who were OO. There was a total of 524 male patients with documented lesion counts - 241 in the combined NER1006 group and 283 in the OSS/2L PEG group. BMI data at the screening visit were available for 237 males in the NER1006 group and 278 males in the OSS/2L PEG group. Males who 
were given NER1006 had a comparable BMI to males who were given OSS/2 L PEG (mean \pm standard deviation [SD]: $29.1 \pm 4.6$ versus $28.9 \pm 5.2 ; P=0.262$ ).

To assess whether combined treatment result trends could be observed also at the individual trial level, all the comparative analyses performed on the combined treatment groups were also repeated within each of the trials MORA and NOCT.

\section{Statistics}

All analyses were carried out using the statistical package $R$ version 4.0.2 (The R Foundation for Statistical Computing, 2020).

The near-identical design of the two randomized phase 3 clinical trials, MORA and NOCT, allowed us to combine the data to perform combined data analysis for NER1006 [21]. Combining OSS and 2 L PEG was reasonable as both have shown similar adequate cleansing success rates with NER1006 but inferior HQ cleansing [15]. OSS and 2 L PEG have also shown similar real-world cleansing performance when using a validated cleansing scale [20].

All statistical comparisons used the two-sided t-test, assuming equal variance, to assess for differences between NER1006 and comparator solutions. As a complement to t-tests, the possibility to more precisely detect male patients with three or more adenomas was assessed using multiple logistic regression. Unlike the t-test, this technique mitigates the risk of variable interactions, and its primary function is to predict clinical outcomes. Multiple regression analyses were performed using the backward elimination method to obtain minimal models that could identify the most important predictors of ADR3 + among 20 clinical and patient characteristics. Odds ratios with a $95 \% \mathrm{Cl}$ were calculated from the coefficients generated by the logistic regression. $P<0.05$ was considered significant.

The detection rates PDR, ADR, rPDR, and rADR were all calculated by dividing the number of patients with one or more lesions detected per patient by the total number of patients in the same cohort. Similarly, ADR3 + and ADR5 + were calculated by dividing the number of patients with three $(A D R 3+)$ or five $(A D R 5+)$ or more adenomas detected per patient by the total number of patients in the same cohort.

MPP and MAP results (MPP + and MAP + for patients with at least one neoplasia) are presented as means with a $(95 \% \mathrm{Cl}$, lower and upper limits).

The attainment of overall HCS Grade AB or A was calculated by dividing the number of patients with either grade by the total number of patients in the same cohort. The number of HQ cleansed colon segments per treatment group was calculated by dividing the total number of colon segments with HCS score 3 to 4 by the total number of HCS colon segments (five per patient) in the same cohort.

\section{Results}

A total of 1028 patients (NER1006 $=513$ and OSS $/ 2$ L PEG = 515) with documented overall and right colon polyp and adenoma counts were included. Patient selection is presented in Supplementary Table 1, and comparable patient characteristics are presented briefly in Supplementary Tables 2 and 3. The in- cluded population of 1028 patients represents $87 \%$ of the combined full analysis set and $93 \%$ of the modified full analysis set (the original primary analysis set).

\section{Lesion detection and cleansing outcomes in males versus females}

As expected, the 524 male patients showed greater polyp and adenoma detection rates versus females on most performed assessments ( $P \leq 0.002$ for all statistically significant comparisons) and numerically greater results on the remaining few ( $>$ Table 1).

The lower rate of detection in females could not be explained by a worse colon cleansing quality, since adequate cleansing rates in females and males were comparable by central readers (93.2\% [479/514] versus 93.0\% [466/501]; $P=$ 0.912 ) and site colonoscopists (94.2\% [484/514] versus $93.4 \%$ [468/501]; $P=0.621$ ). Females had higher rates of HQ success (HCS A) and HQ cleansed segments than males (15.0\% [77) 514] versus $20.2 \%$ [101/501]; $P=0.030$ and $41.6 \%$ [1069/ $2570]$ versus $45.3 \%$ [1134/2505]; $P=0.008)$.

\section{Lesion detection and cleansing outcomes in 00 versus lean men}

Most males (82.3\% [424/515]) with available BMI data were OO ( Table 2). Only 91 males had a BMI <25. OO males were slightly older than lean males (mean \pm SD: $56.5 \pm 10.9$ versus $53.1 \pm 14.2 ; P=0.010)$.

The analysis of $\mathrm{OO}$ versus lean males indicated a greater prevalence of colorectal polyps in the overall and right colon of OO males. The PDR was $57.8 \%$ (245/424) versus $40.7 \%$ (37/91); $P=0.003$, and the rPDR was $27.8 \%(118 / 424)$ vs $13.2 \%(12 / 91)$; $P=0.003$. Similarly, more $O O$ males had at least one adenoma (ADR $=43.4 \%$ [184/424] versus $23.1 \%$ [21/91]; $P<0.001)$ or at least three adenomas (ADR3 $+=11.6 \%$ [49/424] versus $5.5 \%$ [5/91]; $P=0.087$ ) and more right colon adenomas (rMAP= 0.30 [0.23-0.37] versus $0.10[-0.17-0.22] ; P=0.013$ ) compared with the lean males.

The cleansing quality analysis indicated mostly comparable colon cleansing outcomes in OO versus lean males. One exception was the site colonoscopists who recorded more HQ cleansed segments in males with BMI $\geq 25$ than in males with BMI < 25 (85.2\% [1797/2110] versus 79.3\% [353/445]; $P=$ $0.002)$.

Numerically lower MPP +, MAP +, rMPP +, and rMAP+ were noted in the $\mathrm{OO}$ group of males. One patient in the lean males' group (BMI 23.7) had 37 polyps and 37 adenomas. Excluding that patient resulted in an expected greater MPP (1.57 [1.321.81 ] versus $0.74(0.45-1.04) ; P=0.002)$, MAP (0.95 [0.79$1.11]$ versus $0.41[0.19-0.63] ; P=0.002)$, rMPP $(0.44[0.36-$ $0.52]$ versus $0.18[0.05-0.31] ; P=0.003)$, rMAP $(0.30$ [0.23$0.37]$ versus $0.10[-0.02-0.22] ; P=0.007)$, and $\mathrm{MPP}+(2.71$ [2.35-3.07] versus 1.86 [1.29-2.43]; $P=0.041)$ in 00 males versus lean males. The ADR3 + was also greater in the $\mathrm{BMI} \geq 25$ group versus $\mathrm{BMI}<25$, indicating that $\mathrm{BMI} \geq 25$ group indeed had more patients with multiple adenomas than the $\mathrm{BMI}<25$ group. 
- Table 1 Lesion detection and colon cleansing outcomes in males versus females using either NER1006, 2LPEG, or OSS for bowel preparation in the combined MORA and NOCT trials.

\begin{tabular}{|c|c|c|c|c|}
\hline MORA + NOCT combined & Males & Females & Diff M-F & $P$ \\
\hline Patients with lesion counts, $\mathrm{N}$ & 524 & 504 & 20 & NA \\
\hline - Polyps (mean, $95 \% \mathrm{Cl}$ ) & $1.50(1.26-1.79)$ & $0.91(0.76-1.07)$ & 0.59 & $<0.001$ \\
\hline - Adenoma (mean, 95\% Cl) & $0.93(0.74-1.12)$ & $0.50(0.39-0.61)$ & 0.44 & $<0.001$ \\
\hline - rPolyp (mean, 95\% Cl) & $0.45(0.38-0.58)$ & $0.22(0.17-0.28)$ & 0.23 & 0.002 \\
\hline - rAdenoma (mean, $95 \% \mathrm{Cl}$ ) & $0.26(0.20-0.32)$ & $0.14(0.10-0.19)$ & 0.12 & 0.002 \\
\hline - $\operatorname{PDR}(\%, \mathrm{n} / \mathrm{N})$ & $55.2(289 / 524)$ & $38.5(194 / 504)$ & 16.7 & $<0.001$ \\
\hline - $\operatorname{ADR}(\%, \mathrm{n} / \mathrm{N})$ & $40.3(211 / 524)$ & $25.0(126 / 504)$ & 15.3 & $<0.001$ \\
\hline - $\operatorname{ADR3}(\%, \mathrm{n} / \mathrm{N})$ & $10.7(56 / 524)$ & $5.8(29 / 504)$ & 4.9 & 0.004 \\
\hline - $\operatorname{ADR5}(\%, \mathrm{n} / \mathrm{N})$ & $3.4(18 / 524)$ & $2.0(10 / 504)$ & 1.4 & 0.153 \\
\hline - rPDR $(\%, n / N)$ & $25.4(133 / 524)$ & $16.3(82 / 504)$ & 9.1 & $<0.001$ \\
\hline - $\operatorname{rADR}(\%, \mathrm{n} / \mathrm{N})$ & $17.0(89 / 524)$ & $10.3(52 / 504)$ & 6.7 & 0.002 \\
\hline - $\mathrm{MPP}+($ mean, $95 \% \mathrm{Cl})$ & $2.73(2.34-3.12)$ & $2.37(2.06-2.68)$ & 0.36 & 0.196 \\
\hline - $\mathrm{MAP}+($ mean, $95 \% \mathrm{Cl})$ & $2.32(1.91-2.73)$ & $1.99(1.69-2.30)$ & 0.33 & 0.270 \\
\hline - rMPP + (mean, $95 \% \mathrm{Cl})$ & $1.77(1.32-2.27)$ & $1.38(1.16-1.60)$ & 0.40 & 0.193 \\
\hline - rMAP+ (mean, $95 \% \mathrm{Cl})$ & $1.64(1.42-1.86)$ & $1.50(1.16-1.84)$ & 0.14 & 0.465 \\
\hline $\begin{array}{l}\text { Patients with lesion counts and HCS scores by site } \\
\text { colonoscopists and by central readers, N }\end{array}$ & 514 & 501 & 13 & NA \\
\hline - Adequate HCS AB by CR $(\%, n / N)$ & $93.2(479 / 514)$ & $93.0(466 / 501)$ & 0.2 & 0.912 \\
\hline - HQ HCS A by CR $(\%, n / N)$ & $15.0(77 / 514)$ & $20.2(101 / 501)$ & -5.2 & 0.030 \\
\hline - HQ scores by CR $(\%, \mathrm{n} / \mathrm{N})$ & $41.6(1069 / 2570)$ & $45.3(1134 / 2505)$ & -3.7 & 0.008 \\
\hline - Adequate HCS AB by SC $(\%, n / N)$ & $94.2(484 / 514)$ & $93.4(468 / 501)$ & 0.8 & 0.621 \\
\hline - HQ HCS A by SC $(\%, n / N)$ & $67.9(349 / 514)$ & $65.7(329 / 501)$ & 2.2 & 0.451 \\
\hline - HQ scores by SC (\%, n/N) & $84.0(2184 / 2600)$ & $82.4(2060 / 2500)$ & 1.6 & 0.127 \\
\hline
\end{tabular}

PEG, polyethylene glycol; OSS, oral sulfate solution; PDR, polyp detection rate; MPP, mean overall polyps per patient; MAP, mean adenomas per patient; ; rMPP mean number of polyps in the right colon; rMAP, mean number of adenomas in the right colon; ADR, adenoma detection rate; rADP, adenoma detection rate in the right colon; rPDR, polyp detection rate in the right colon; HCS, Harefield Cleansing Scale; HQ high-quality.

\section{Lesion detection and cleansing outcomes with NER 1006 vs OSS/2 L PEG}

Given the importance of both age and BMI for adenoma detection, it is notable that these two parameters were comparable between the two combined study groups; NER1006 versus OSS/2L PEG: Age (mean \pm SD: $56.7 \pm 11.3$ versus $56.4 \pm 10.6$ years; $P=0.379$ ) and $B M I$ (mean \pm SD: $30.2 \pm 4.1$ versus $30.2 \pm$ 4.8; $P=0.456$ ) (Supplementary Table 3).

The combined group analysis in all males showed a numerical improvement in polyp or adenoma detection with NER1006 versus OSS/2L PEG in all performed analyses ( $\downarrow$ Table 3 ). NER1006 also achieved improvements in the MPP (1.75 [1.282.21 ] versus 1.30 [1.08-1.52]; $P=0.072)$ and MAP (1.14 [0.771.51 ] versus 0.76 [0.60-0.92]; $P=0.049)$. In polyp- or adenoma-positive patients, NER1006 also helped detect more lesions than OSS/2 L PEG (MPP+: 3.17 [2.403.93] versus 2.35 [2.04-
2.67]; $P=0.041$ and $\mathrm{MAP}+: 2.78$ [1.97-3.58] versus 1.91 [1.61-2.21]; $P=0.037)$. NER1006 enabled greater detection rates of patients with at least three (ADR3: $13.7 \%$ [33/241] versus $8.1 \%$ [23/283]; $P=0.040$ ) or at least five (ADR5: $5.0 \%$ [12/ 241 ] versus $2.1 \%$ [6/283]; $P=0.074$ ) adenomas. There was a $68 \%$ greater detection rate of patients with at least three adenomas. The NER1006 group in NOCT had the outlier with 37 adenomas. Excluding that outlier, MPP (1.60 [1.23-1.97] versus 1.30 [1.08-1.52]; $P=0.149)$, ADR5 (4.6\% [11/240] versus $2.1 \%[6 / 283] ; P=0.114)$, MAP (0.99 [0.76-1.22] versus 0.76 [0.60-0.92]; $P=0.089)$, ADR3 (13.3\% [32/240] versus $8.1 \%$ [23/283]; $P=0.053)$, and MPP + (2.91 [2.33-3.49] versus 2.35 [2.04-2.67]; $P=0.083)$ remained numerically greater with NER1006 versus OSS/2 L PEG, while MAP+ (2.43 [2.01-2.84] versus $1.91[1.61-2.21] ; P=0.042)$ remained greater with NER1006. 
- Table 2 Lesion detection and cleansing quality in $\mathrm{OO}$ males (BMI 225$)$ versus lean males $(\mathrm{BMI}<25)$ using either NER1006, 2LPEG, or OSS for bowel preparation in the combined MORA and NOCT trials.

\begin{tabular}{|c|c|c|c|c|}
\hline \multirow[t]{2}{*}{ MORA + NOCT combined } & Males with & Males with & \multirow{2}{*}{$\begin{array}{l}\text { Diff } \\
M-F\end{array}$} & \multirow[t]{2}{*}{$P$} \\
\hline & $\mathrm{BMI} \geq 25 \mathrm{~kg} / \mathrm{m}^{2}$ & $\mathrm{BMI}<25 \mathrm{~kg} / \mathrm{m}^{2}$ & & \\
\hline $\begin{array}{l}\text { Patients with lesion counts and BMI data } \\
\text { at screening visit, } N\end{array}$ & 424 & 91 & 333 & NA \\
\hline - MPP (mean, $95 \%$ Cl) & $1.57(1.32-1.81)$ & $1.14(0.30-1.99)$ & 0.43 & 0.202 \\
\hline - MAP (mean, $95 \% \mathrm{Cl}$ ) & $0.95(0.79-1.11)$ & $0.81(-0.01-1.64)$ & 0.15 & 0.590 \\
\hline - rMPP (mean, 95\% Cl) & $0.44(0.36-0.52)$ & $0.51(-0.16-1.17)$ & -0.07 & 0.698 \\
\hline - rMAP (mean, $95 \% \mathrm{Cl}$ ) & $0.30(0.23-0.37)$ & $0.10(-0.02-0.22)$ & 0.20 & 0.013 \\
\hline - $\operatorname{PDR}(\%, n / N)$ & $57.8(245 / 424)$ & $40.7(37 / 91)$ & 17.12 & 0.003 \\
\hline - $\operatorname{ADR}(\%, \mathrm{n} / \mathrm{N})$ & $43.4(184 / 424)$ & $23.1(21 / 91)$ & 20.32 & $<0.001$ \\
\hline - $\operatorname{ADR3}(\%, \mathrm{n} / \mathrm{N})$ & $11.6(49 / 424)$ & $5.5(5 / 91)$ & 6.06 & 0.087 \\
\hline - $\operatorname{ADR5}(\%, \mathrm{n} / \mathrm{N})$ & $3.5(15 / 424)$ & $3.3(3 / 91)$ & 0.24 & 0.910 \\
\hline - $r$ PDR $(\%, n / N)$ & $27.8(118 / 424)$ & $13.2(12 / 91)$ & 14.64 & 0.003 \\
\hline - $\operatorname{rADR}(\%, \mathrm{n} / \mathrm{N})$ & $19.6(83 / 424)$ & $5.5(5 / 91)$ & 14.08 & 0.001 \\
\hline - MPP + (mean, $95 \% \mathrm{Cl})$ & $2.71(2.35-3.07)$ & $2.81(0.81-4.82)$ & -0.10 & 0.868 \\
\hline - $\mathrm{MAP}+($ mean, $95 \% \mathrm{Cl})$ & $2.20(1.92-2.47)$ & $3.52(-0.03-7.08)$ & -1.32 & 0.059 \\
\hline - rMPP + (mean, $95 \% \mathrm{Cl})$ & $1.57(1.40-1.73)$ & $3.83(-1.45-9.12)$ & -2.27 & 0.005 \\
\hline - rMAP+(mean, $95 \% \mathrm{Cl})$ & $1.64(1.42-1.85)$ & $1.80(-0.42-4.02)$ & -0.16 & 0.735 \\
\hline $\begin{array}{l}\text { Patients with lesion counts, BMI data at } \\
\text { screening visit and HCS scores by site colo- } \\
\text { noscopists and by central readers, } \mathrm{N}\end{array}$ & 416 & 89 & 327 & NA \\
\hline - Adequate HCS AB by CR $(\%, n / N)$ & $93.3(388 / 416)$ & $92.1(82 / 89)$ & 1.2 & 0.703 \\
\hline - HQ HCS A by CR $(\%, n / N)$ & $16.1(67 / 416)$ & $11.2(10 / 89)$ & 4.9 & 0.247 \\
\hline - $\mathrm{HQ}$ scores by CR $(\%, \mathrm{n} / \mathrm{N})$ & $42.2(877 / 2080)$ & $39.8(177 / 445)$ & 2.4 & 0.354 \\
\hline - Adequate HCS AB by SC $(\%, n / N)$ & $94.5(393 / 416)$ & $92.1(82 / 89)$ & 2.4 & 0.398 \\
\hline - HQ HCS A by SC $(\%, n / N)$ & $69.7(290 / 416)$ & $62.9(56 / 89)$ & 6.8 & 0.211 \\
\hline - HQ scores by SC $(\%, n / N)$ & $85.2(1797 / 2110)$ & $79.3(353 / 445)$ & 5.9 & 0.002 \\
\hline
\end{tabular}

PEG, polyethylene glycol; OSS, oral sulfate solution; PDR, polyp detection rate; MPP, mean overall polyps per patient; MAP, mean adenomas per patient; rMPP mean number of polyps in the right colon; rMAP, mean number of adenomas in the right colon; ADR, adenoma detection rate; rADP, adenoma detection rate in the right colon; rPDR, polyp detection rate in the right colon; HCS, Harefield Cleansing Scale; HQ high-quality.

In $\mathrm{OO}$ males, the MAP and MAP+ were greater with NER1006 versus OSS/2L PEG (1.11 [0.85-1.38] versus $0.81[0.63-1.00]$; $P=0.065$ and $2.54[2.08-3.00]$ versus $1.89[1.57-2.21] ; P=$ 0.019 ) ( $\checkmark$ Table 4). In polyp-positive patients, more polyps were detected in patients who received NER1006 (MPP+: 3.12 [2.45-3.79] versus $2.4[2.028-2.709] ; P=0.040$ and $r M P P+$ : $1.72[1.44-2.00]$ versus 1.43 [1.24-1.61]; $P=0.077$ ). The relative detection rate of patients with multiple adenomas was improved by $79 \%$ for ADR3 (15.1\% [30/199] versus 8.4\% [19/ $225] ; P=0.033)$ and by $311 \%$ for ADR5 (5.5\% [11/199] versus $1.8 \%[4 / 225] ; P=0.037)$.

In the per-trial analysis in all males, NER1006 showed a numerically improved polyp or adenoma detection in all performed analyses versus 2L PEG. Improvements with NER1006 versus 2 L PEG were observed for the rMPP $(0.56[0.35-0.77]$ versus 0.30 [0.16-0.43]; $P=0.031)$, rPDR (30.3\% [33/109] versus $17.2 \%$ [23/134]; $P=0.016)$, and rADR (18.3\% [20/109] versus $9.7 \%[13 / 134] ; P=0.051)$. NER1006 also showed a numerically improved polyp or adenoma detection in 9 out of 14 performed analyses versus OSS. NER1006 achieved a greater ADR5 + (5.3\% [7/132] versus $1.3 \%[2 / 149] ; P=0.060)$ and a greater MAP + (3.23 [1.79-4.66] versus 1.85 [1.51-2.18]; $P=$ 0.041 ) than OSS. While five of the analyses (PDR, ADR, rPDR, rADR, and rMAP) showed numerical improvements with OSS versus NER1006, none of these analyses reached statistical significance. Despite excluding the lean male outlier with 37 adenomas, NER1006 continued to demonstrate an improvement in 
- Table 3 Lesion detection in all male patients, per trial, and in the combined treatment groups.

\section{MORA}

- Patients, N

- MPP (mean, $95 \% \mathrm{Cl})$

- $\operatorname{MAP}($ mean, $95 \% \mathrm{Cl})$

- rMPP (mean, $95 \% \mathrm{Cl}$ )

- $\operatorname{rMAP}($ mean, $95 \% \mathrm{Cl})$

- $\operatorname{PDR}(\%, \mathrm{n} / \mathrm{N})$

- $\operatorname{ADR}(\%, \mathrm{n} / \mathrm{N})$

- $\operatorname{ADR3}(\%, \mathrm{n} / \mathrm{N})$

- $\operatorname{ADR5}(\%, \mathrm{n} / \mathrm{N})$

- $\operatorname{rPDR}(\%, n / N)$

- $\operatorname{rADR}(\%, \mathrm{n} / \mathrm{N})$

- $\mathrm{MPP}+($ mean, $95 \% \mathrm{Cl})$

- $\mathrm{MAP}+($ mean, $95 \% \mathrm{Cl})$

- rMPP+(mean, $95 \% \mathrm{Cl})$

- rMAP+ (mean, $95 \% \mathrm{Cl})$

\section{NOCT}

- Patients,N

- $\operatorname{MPP}($ mean, $95 \% \mathrm{Cl})$

- MAP (mean, $95 \% \mathrm{Cl}$ )

- $\operatorname{rMPP}($ mean, $95 \% \mathrm{Cl}$ )

- $\operatorname{rMAP}($ mean, $95 \% \mathrm{Cl}$ )

- $\operatorname{PDR}(\%, \mathrm{n} / \mathrm{N})$

- $\operatorname{ADR}(\%, n / N)$

- $\operatorname{ADR3}(\%, n / N)$

- $\operatorname{ADR5}(\%, \mathrm{n} / \mathrm{N})$

- $\operatorname{rPDR}(\%, \mathrm{n} / \mathrm{N})$

- $\operatorname{rADR}(\%, n / N)$

- $\mathrm{MPP}+($ mean, $95 \% \mathrm{Cl})$

- $\mathrm{MAP}+($ mean, $95 \% \mathrm{Cl})$

- $r M P P+($ mean, $95 \% \mathrm{Cl})$

- $r M A P+(m e a n, 95 \% \mathrm{Cl})$

MORA/NOCT combined

- Patients, N

- MPP (mean, 95\% Cl)

- MAP (mean, $95 \% \mathrm{Cl}$ )

- $\operatorname{rMPP}($ mean, $95 \% \mathrm{Cl})$

- $\operatorname{rMAP}($ mean, $95 \% \mathrm{Cl}$ )

- $\operatorname{PDR}(\%, \mathrm{n} / \mathrm{N})$

- $\operatorname{ADR}(\%, \mathrm{n} / \mathrm{N})$

- $\operatorname{ADR3}(\%, \mathrm{n} / \mathrm{N})$
NER1006

109

$1.90(1.20-2.60)$

$0.95(0.63-1.27)$

$0.56(0.35-0.77)$

$0.32(0.16-0.48)$

$56.0(61 / 109)$

$42.2(46 / 109)$

$11.9(13 / 109)$

$4.6(5 / 109)$

$30.3(33 / 109)$

$18.3(20 / 109)$

$3.39(2.26-4.52)$

$2.26(1.68-2.84)$

$1.85(1.41-2.28)$

$1.95(1.28-2.62)$

NER1006

132

$1.62(0.99-2.25)$

$1.30(0.67-1.92)$

$0.57(0.11-1.03)$

$0.27(0.16-0.37)$

$54.5(72 / 132)$

$40.2(53 / 132)$

$15.2(20 / 132)$

$5.3(7 / 132)$

$24.2(32 / 132)$

$18.9(25 / 132)$

2.97 (1.91-4.04)

$3.23(1.79-4.66)$

$2.34(0.51-4.18)$

$1.48(1.19-1.78)$

\section{NER1006}

241

$1.75(1.28-2.21)$

$1.14(0.77-1.51)$

$0.56(0.30-0.83)$

$0.29(0.20-0.38)$

$55.2(133 / 241)$

41.1 (99/241)

$13.7(33 / 241)$
2L PEG

134

$1.35(1.01-1.69)$

$0.69(0.44-0.93)$

$0.30(0.16-0.43)$

$0.18(0.07-0.29)$

$50.7(68 / 134)$

$34.3(46 / 134)$

$6.7(9 / 134)$

$3.0(4 / 134)$

$17.2(23 / 134)$

$9.7(13 / 134)$

$2.66(2.16-3.16)$

$2.00(1.45-2.55)$

$1.74(1.27-2.21)$

$1.92(1.13-2.72)$

OSS

149

$1.25(0.96-1.54)$

$0.82(0.61-1.03)$

$0.40(0.29-0.52)$

$0.29(0.19-0.39)$

$59.1(88 / 149)$

$44.3(66 / 149)$

$9.4(14 / 149)$

$1.3(2 / 149)$

$30.2(45 / 149)$

$20.8(31 / 149)$

$2.11(1.71-2.52)$

$1.85(1.51-2.18)$

$1.33(1.14-1.53)$

$1.45(1.19-1.71)$

2 L PEG/OSS

283

$1.30(1.08-1.52)$

$0.76(0.60-0.92)$

$0.35(0.27-0.44)$

$0.24(0.16-0.31)$

$55.1(156 / 283)$

$39.6(112 / 283)$

$8.1(23 / 283)$
Difference

-

0.548

0.267

0.261

0.142

5.3

7.9

5.2

1.6

13.1

8.6

0.732

0.261

0.109

0.027

Difference

$-$

0.373

0.476

0.270

0.136

0.166

0.468

$-0.023$

0.755

$-4.5$

0.447

$-4.1$

0.485

5.8

0.141

4.0

0.060

$-6.0$

0.265

$-1.9$

0.697

0.859

0.109

1.378

0.041

1.010

0.191

0.028

0.884

Difference

P

-

0.450

0.072

0.385

0.049

0.211

0.116

0.053

0.377

0.001

0.989

1.5

0.727

5.6

0.040 


\begin{tabular}{|c|c|c|c|c|}
\hline - $\operatorname{ADR} 5(\%, \mathrm{n} / \mathrm{N})$ & $5.0(12 / 241)$ & $2.1(6 / 283)$ & 2.9 & 0.074 \\
\hline - $\operatorname{rPDR}(\%, \mathrm{n} / \mathrm{N})$ & $27.0(65 / 241)$ & $24.0(68 / 283)$ & 3.0 & 0.441 \\
\hline - $\operatorname{rADR}(\%, \mathrm{n} / \mathrm{N})$ & $18.7(45 / 241)$ & $15.5(44 / 283)$ & 3.1 & 0.343 \\
\hline - $\mathrm{MPP}+($ mean, $95 \% \mathrm{Cl})$ & $3.17(2.40-3.93)$ & $2.35(2.04-2.67)$ & 0.812 & 0.041 \\
\hline - $\mathrm{MAP}+($ mean, $95 \% \mathrm{Cl})$ & $2.78(1.97-3.58)$ & $1.91(1.61-2.21)$ & 0.867 & 0.037 \\
\hline - $r M P P+(m e a n, 95 \% \mathrm{Cl})$ & $2.09(1.19-3.00)$ & $1.47(1.27-1.67)$ & 0.621 & 0.174 \\
\hline - rMAP+ (mean, $95 \% \mathrm{Cl})$ & $1.69(1.36-2.02)$ & $1.59(1.30-1.88)$ & 0.098 & 0.655 \\
\hline
\end{tabular}

PEG, polyethylene glycol; OSS, oral sulfate solution; PDR, polyp detection rate; MPP, mean overall polyps per patient; MAP, mean adenomas per patient; rMPP mean number of polyps in the right colon; rMAP, mean number of adenomas in the right colon; HCS, Harefield Cleansing Scale; HQ high-quality.

MAP + in all males versus OSS $(2.58[1.97-3.19]$ versus 1.85 [1.51-2.18]; $P=0.029$ ).

In $\mathrm{OO}$ males, the per-trial analysis demonstrated numerical improvements in most lesion detection measures in both the MORA trial and the NOCT trial. Several of the improvements with NER1006 (ADR3, rPDR, rADR, MPP+, rMPP, and rMAP) had statistically significant P-values. PDR was numerically only improved with 2 L PEG $(P=0.557)$. NER1006 achieved numerically improved detection rates in eight of 14 performed analyses versus OSS. The MAP+reached an appreciable difference (2.61 [1.99-3.23] versus $1.88[1.51-2.25] ; P=0.039)$ with NER1006 compared with OSS. None of the six numerically improved lesion detections with OSS versus NER1006 (PDR, ADR, rPDR, IADR, rMPP, rMAP) reached statistical significance.

Since combined cleansing data have already been published for NER1006 versus OSS/2L PEG $[15,16]$, the cleansing outcomes were only assessed per trial.

When cleansing was assessed by site colonoscopists, adequate success rates were comparable between treatments in both the MORA and the NOCT trial ( $\triangleright$ Table 5 ). HQ success (HCS Grade A) rates were greater with NER1006 versus 2 L PEG in all males (74.3\% [81/109] versus $50.0 \%$ [67/134]; $P<0.001)$ and in 00 males (79.5\% [66/83] versus $50.5 \%$ [50/99]; $P<$ 0.001). The number of HQ cleansed segments (HSC score 3-4; stool-free) was greater with NER1006 than 2L PEG in both all males and in 00 males $(89.8 \%$ [485/540] versus $72.4 \%$ [478/ 660]; $P<0.001$ and $90.8 \%[377 / 415]$ versus $74.1 \%$ [363/490]; $P<0.001)$. The number of $\mathrm{HQ}$ cleansed segments was also greater with NER1006 than OSS in both all males and in OO males $(89.9 \%$ [589/655] versus $84.8 \%$ [632/745]; $P=0.004$ and $90.1 \%[518 / 575]$ versus $85.6 \%$ [539/630]; $P=0.017$ ).

The stricter cleansing assessment by central readers confirmed the improved HQ cleansing with NER1006 versus 2L PEG or OSS.NER1006 attained more HQ cleansed segments than 2 L PEG in both all males and in OO males (49.1\% [265/ 540] versus $31.7 \%$ [211/665]; $P<0.001$ and $48.9 \%$ [203/415] versus $32.7 \%$ [160/490]; $P<0.001$ ). Similarly, in both all males and in $\mathrm{OO}$ males, NER1006 attained greater HQ success (HCS Grade A) rates than OSS (18.8\% [24/128] versus $9.7 \%$ [14/ 145]; $P=0.030$ and $20.5 \%$ [23/112] versus $10.6 \%$ [13/123]; $P=$ 0.017). Finally, NER1006 also attained more HQ cleansed segments per treatment group than OSS in both all males $(47.5 \%$
[304/640] versus $39.9 \%[289 / 725] ; P=0.004)$ and in 00 males (47.5\% [266/560] versus $40.3 \%$ [248/615]; $P=0.013)$.

\section{Multiple logistic regression analysis}

Using multiple regression analyses, we found that age (odds ratio $=1.049, \quad P=0.002 ;$ odds ratio $=1.056, \quad P=0.002)$ and NER1006 (odds ratio $=1.902, P=0.037$; odds ratio $=2.049, P=$ 0.030 ) were associated with increased ADR3 + score in the two groups-all males and males with $\mathrm{BMI} \geq 25$, respectively ( $\bullet$ Table 6). However, the association between age and NER1006 was not significant for the ADR5 + score for all males or males with $\mathrm{BMI} \geq 25$.

\section{Discussion}

In this post hoc analysis of two randomized phase 3 clinical trials, we demonstrated that $1 \mathrm{~L}$ NER1006 could help endoscopists detect more male patients with multiple adenomas and that this improved detection of patients at increased risk for poor cleansing and colorectal adenomas and colorectal cancer was associated with an improved HQ colon cleansing.

We confirmed that the combined male study populations in the MORA and NOCT trials had more polyps and adenomas than females and that the higher prevalence of neoplasia in males was not attributable to poorer cleansing in females.

The $\mathrm{OO}$ males with $\mathrm{BMI} \geq 25$ showed more colorectal polyps and adenomas than lean males with a $\mathrm{BMI}<25$. This finding is consistent with the literature reports mentioning high BMI as a risk factor for colorectal adenomas. It was encouraging to learn that $\mathrm{OO}$ males had cleansing outcomes, which were at least as good as those in lean males.

We found an improved detection of colorectal polyps and adenomas with NER1006 versus OSS/2 L PEG, as well as consistent improvement in HQ cleansing with NER 1006 versus OSS/2 L PEG in both analyzed trials. NER1006 attained numerically improved lesion detection on most analyzed parameters within each trial and demonstrated improvements on several in the combined and in the per-trial analyses. While occasional detection rates numerically favored the comparator, none of those rates reached statistical significance. These results, strengthened by the multiple logistic regression analysis, therefore, in- 
- Table4 Lesion detection in $\mathrm{OO}$ male patients, per trial, and in the combined treatment groups.

\section{MORA}

- Patients, N

- MPP (mean, $95 \% \mathrm{Cl})$

- $\operatorname{MAP}($ mean, $95 \% \mathrm{Cl})$

- rMPP (mean, $95 \% \mathrm{Cl}$ )

- $\operatorname{rMAP}($ mean, $95 \% \mathrm{Cl})$

- $\operatorname{PDR}(\%, \mathrm{n} / \mathrm{N})$

- $\operatorname{ADR}(\%, n / N)$

- $\operatorname{ADR3}(\%, \mathrm{n} / \mathrm{N})$

- $\operatorname{ADR5}(\%, \mathrm{n} / \mathrm{N})$

- $\operatorname{rPDR}(\%, n / N)$

- $\operatorname{rADR}(\%, \mathrm{n} / \mathrm{N})$

- $\mathrm{MPP}+($ mean, $95 \% \mathrm{Cl})$

- $\mathrm{MAP}+($ mean, $95 \% \mathrm{Cl})$

- rMPP+(mean, $95 \% \mathrm{Cl})$

- rMAP+ (mean, $95 \% \mathrm{Cl})$

NOCT

- Patients, N

- MPP (mean, $95 \% \mathrm{Cl}$ )

- MAP (mean, $95 \% \mathrm{Cl}$ )

- $\operatorname{rMPP}($ mean, $95 \% \mathrm{Cl}$ )

- $\operatorname{rMAP}($ mean, $95 \% \mathrm{Cl}$ )

- $\operatorname{PDR}(\%, \mathrm{n} / \mathrm{N})$

- $\operatorname{ADR}(\%, \mathrm{n} / \mathrm{N})$

- $\operatorname{ADR3}(\%, \mathrm{n} / \mathrm{N})$

- $\operatorname{ADR5}(\%, n / N)$

- $\operatorname{rPDR}(\%, \mathrm{n} / \mathrm{N})$

- $\operatorname{rADR}(\%, n / N)$

- $\mathrm{MPP}+($ mean, $95 \% \mathrm{Cl})$

- $\mathrm{MAP}+($ mean, $95 \% \mathrm{Cl})$

- rMPP + (mean, $95 \% \mathrm{Cl})$

- rMAP+(mean, $95 \% \mathrm{Cl})$

MORA/NOCT combined

- Patients, N

- $\operatorname{MPP}($ mean, $95 \% \mathrm{Cl}$ )

- MAP (mean, $95 \% \mathrm{Cl}$ )

- $\operatorname{rMPP}($ mean, $95 \% \mathrm{Cl})$

- rMAP (mean, $95 \% \mathrm{Cl}$ )

- $\operatorname{PDR}(\%, \mathrm{n} / \mathrm{N})$

- $\operatorname{ADR}(\%, \mathrm{n} / \mathrm{N})$

- $\operatorname{ADR3}(\%, \mathrm{n} / \mathrm{N})$

\section{NER1006}

83

$2.12(1.21-3.03)$

$1.06(0.66-1.46)$

$0.65(0.39-0.91)$

$0.39(0.18-0.60)$

$54.2(45 / 83)$

$43.4(36 / 83)$

$13.3(11 / 83)$

$6.0(5 / 83)$

$32.5(27 / 83)$

$20.5(17 / 83)$

$3.91(2.41-5.41)$

$2.44(1.73-3.16)$

$2.00(1.49-2.51)$

$2.12(1.35-2.89)$

NER1006

116

$1.49(1.12-1.86)$

$1.15(0.79-1.50)$

$0.38(0.25-0.51)$

$0.30(0.18-0.42)$

$57.8(67 / 116)$

$44.0(51 / 116)$

$16.4(19 / 116)$

$5.2(6 / 116)$

$25.9(30 / 116)$

$21.6(25 / 116)$

$2.58(2.08-3.08)$

$2.61(1.99-3.23)$

$1.47(1.21-1.72)$

$1.48(1.19-1.78)$

\section{NER1006}

199

$1.75(1.32-2.19)$

$1.11(0.85-1.38)$

$0.49(0.36-0.63)$

$0.34(0.23-0.45)$

$56.3(112 / 199)$

43.7 (87/199)

15.1 (30/199)
2L PEG

99

1.44 (1.05-1.84)

$0.73(0.44-1.02)$

$0.30(0.16-0.45)$

$0.18(0.06-0.30)$

54.5 (54/99)

$38.4(38 / 99)$

6.1 (6/99)

$2.0(2 / 99)$

$19.2(19 / 99)$

$11.1(11 / 99)$

$2.65(2.11-3.19)$

$1.89(1.29-2.50)$

$1.58(1.18-1.98)$

$1.72(1.05-2.41)$

OSS

126

1.37 (1.03-1.70)

$0.88(0.64-1.12)$

$0.45(0.32-0.58)$

$0.33(0.21-0.45)$

$62.7(79 / 126)$

$46.8(59 / 126)$

$10.3(13 / 126)$

$1.6(2 / 126)$

$33.3(42 / 126)$

$23.8(30 / 126)$

$2.18(1.73-2.62)$

$1.88(1.51-2.25)$

$1.36(1.15-1.56)$

$1.47(1.19-1.74)$

2LPEG/OSS

225

$1.40(1.15-1.65)$

$0.81(0.63-1.00)$

0.39 (0.29-0.48)

$0.27(0.18-0.35)$

$59.1(133 / 225)$

$43.1(97 / 225)$

$8.4(19 / 225)$
Difference

-

0.676

0.333

0.348

0.204

$-0.300$

5.0

7.2

4.0

13.3

9.4

1.263

0.550

0.421

0.390

Difference

$-$

0.126

0.266

$-0.073$

$-0.032$

$-0.049$

$-0.029$

6.1

3.6

$-7.5$

$-2.3$

0.405

0.726

0.110

0.013

Difference

$-$

0.354

0.298

0.105

0.070

$-2.8$

0.600

6.6

0.033 


\begin{tabular}{|c|c|c|c|c|}
\hline - $\operatorname{ADR5}(\%, \mathrm{n} / \mathrm{N})$ & $5.5(11 / 199)$ & $1.8(4 / 225)$ & 3.7 & 0.037 \\
\hline - $\operatorname{rPDR}(\%, \mathrm{n} / \mathrm{N})$ & $28.6(57 / 199)$ & $27.1(61 / 225)$ & 1.5 & 0.726 \\
\hline - $\operatorname{rADR}(\%, \mathrm{n} / \mathrm{N})$ & $21.1(42 / 199)$ & $18.2(41 / 225)$ & 2.9 & 0.456 \\
\hline - $\mathrm{MPP}+($ mean, $95 \% \mathrm{Cl})$ & $3.12(2.45-3.79)$ & $2.37(2.028-2.709)$ & 0.748 & 0.040 \\
\hline - $\mathrm{MAP}+($ mean, $95 \% \mathrm{Cl})$ & $2.54(2.08-3.00)$ & $1.89(1.57-2.21)$ & 0.653 & 0.019 \\
\hline - $\mathrm{rMPP}+($ mean, $95 \% \mathrm{Cl})$ & $1.72(1.44-2.00)$ & $1.43(1.24-1.61)$ & 0.293 & 0.077 \\
\hline - $\mathrm{rMAP}+($ mean, $95 \% \mathrm{Cl})$ & $1.74(1.39-2.09)$ & $1.54(1.28-1.79)$ & 0.201 & 0.353 \\
\hline
\end{tabular}

PEG, polyethylene glycol; OSS, oral sulfate solution; PDR, polyp detection rate; MPP, mean overall polyps per patient; MAP, mean adenomas per patient; rMPP mean number of polyps in the right colon; rMAP, mean number of adenomas in the right colon; HCS, Harefield Cleansing Scale; HQ high-quality.

- Table 5. Adequate-quality and $\mathrm{HQ}$ colon cleansing in male patients, per trial, assessed by both site colonoscopists (as in real-world practice) and strictly by central readers.

\begin{tabular}{|c|c|c|c|c|c|c|c|c|}
\hline \multirow{3}{*}{$\begin{array}{l}\text { Clinical trial } \\
\text { cleansing quality assessor } \\
\text { Treatment }\end{array}$} & \multicolumn{2}{|l|}{ MORA } & \multicolumn{2}{|l|}{ NOCT } & \multicolumn{2}{|l|}{ MORA } & \multicolumn{2}{|l|}{ NOCT } \\
\hline & \multicolumn{4}{|c|}{ Site colonoscopists } & \multicolumn{4}{|c|}{ Central readers } \\
\hline & NER1006 & 2L PEG & NER1006 & oss & NER1006 & 2L PEG & NER1006 & oss \\
\hline Male patients, $\mathrm{N}$ & 109 & 134 & 131 & 149 & 108 & 133 & 128 & 145 \\
\hline \multirow[t]{2}{*}{ HCS Grade AB \%, $(n / N)$} & $\begin{array}{l}97.2(106 / \\
109)\end{array}$ & $\begin{array}{l}92.5(124 / \\
134)\end{array}$ & $\begin{array}{l}93.1(122 / \\
131)\end{array}$ & $\begin{array}{l}94.6(141 / \\
149)\end{array}$ & $\begin{array}{l}96.3(104 / \\
108)\end{array}$ & $\begin{array}{l}93.2(124 / \\
133)\end{array}$ & $\begin{array}{l}90.6(116 / \\
128)\end{array}$ & $\begin{array}{l}93.1(135 / \\
145)\end{array}$ \\
\hline & \multicolumn{2}{|l|}{$P=0.106$} & \multicolumn{2}{|l|}{$P=0.601$} & \multicolumn{2}{|l|}{$P=0.297$} & \multicolumn{2}{|l|}{$P=0.455$} \\
\hline \multirow[t]{2}{*}{ HCS Grade A \%, (n/N) } & $\begin{array}{l}74.3(81 / \\
109)\end{array}$ & $\begin{array}{l}50.0(67 / \\
134)\end{array}$ & $\begin{array}{l}75.6(99 / \\
131)\end{array}$ & $\begin{array}{l}72.5(108 / \\
149)\end{array}$ & $\begin{array}{l}20.4(22 / \\
108)\end{array}$ & $\begin{array}{l}12.8(17 / \\
133)\end{array}$ & $\begin{array}{l}18.8(24 / \\
128)\end{array}$ & $\begin{array}{l}9.7(14 / \\
145)\end{array}$ \\
\hline & \multicolumn{2}{|l|}{$P<0.001$} & \multicolumn{2}{|l|}{$P=0.559$} & \multicolumn{2}{|l|}{$P=0.113$} & \multicolumn{2}{|l|}{$P=0.030$} \\
\hline \multirow[t]{2}{*}{ Number of HQ segments \% } & $\begin{array}{l}89.8(485 / \\
540)\end{array}$ & $\begin{array}{l}72.4(478 / \\
660)\end{array}$ & $\begin{array}{l}89.9(589 / \\
655)\end{array}$ & $\begin{array}{l}84.8(6321 \\
745)\end{array}$ & $\begin{array}{l}49.1(265 / \\
540)\end{array}$ & $\begin{array}{l}31.7(211 / \\
665)\end{array}$ & $\begin{array}{l}47.5(304 / \\
640)\end{array}$ & $\begin{array}{l}39.9(289 / \\
725)\end{array}$ \\
\hline & \multicolumn{2}{|l|}{$P<0.001$} & \multicolumn{2}{|l|}{$P=0.004$} & \multicolumn{2}{|l|}{$P<0.001$} & \multicolumn{2}{|l|}{$P=0.004$} \\
\hline $\mathrm{OO}$ males, $\mathrm{N}$ & 83 & 99 & 115 & 126 & 83 & 98 & 112 & 123 \\
\hline HCS Grade AB \%, (n/N) & $\begin{array}{l}98.8(82 / \\
83)\end{array}$ & $\begin{array}{l}91.9(91 / \\
99)\end{array}$ & $\begin{array}{l}93.0(107 / \\
115)\end{array}$ & $\begin{array}{l}95.2(120 / \\
126)\end{array}$ & $\begin{array}{l}96.4(80 / \\
83)\end{array}$ & $\begin{array}{l}92.9(91 / / \\
98)\end{array}$ & $\begin{array}{l}91.1(102 / \\
112)\end{array}$ & $\begin{array}{l}93.5(115 / \\
123)\end{array}$ \\
\hline$P$ value & \multicolumn{2}{|l|}{$P=0.033$} & \multicolumn{2}{|l|}{$P=0.469$} & \multicolumn{2}{|l|}{$P=0.303$} & \multicolumn{2}{|l|}{$P=0.756$} \\
\hline \multirow[t]{2}{*}{ HCS Grade A \%, (n/N) } & $\begin{array}{l}79.5(66 / \\
83)\end{array}$ & $\begin{array}{l}50.5(50 / \\
99)\end{array}$ & $\begin{array}{l}75.7(87 / \\
115)\end{array}$ & $\begin{array}{l}73.8(93 / \\
126)\end{array}$ & $\begin{array}{l}21.7(18 / \\
83)\end{array}$ & $\begin{array}{l}13.3(13 / \\
98)\end{array}$ & $\begin{array}{l}20.5(23 / \\
112)\end{array}$ & $\begin{array}{l}10.6(13 / \\
123)\end{array}$ \\
\hline & \multicolumn{2}{|l|}{$P<0.001$} & \multicolumn{2}{|l|}{$P=0.744$} & \multicolumn{2}{|l|}{$P=0.136$} & \multicolumn{2}{|l|}{$P=0.017$} \\
\hline Number of HQ segments \% & $\begin{array}{l}90.8(377 / \\
415)\end{array}$ & $\begin{array}{l}74.1(363 / \\
490)\end{array}$ & $\begin{array}{l}90.1(518 / \\
575)\end{array}$ & $\begin{array}{l}85.6(539 / \\
630)\end{array}$ & $\begin{array}{l}48.9(203 / \\
415)\end{array}$ & $\begin{array}{l}32.7(160 / \\
490)\end{array}$ & $\begin{array}{l}47.5(266 / \\
560)\end{array}$ & $\begin{array}{l}40.3(248 / \\
615)\end{array}$ \\
\hline$P$ value & \multicolumn{2}{|l|}{$P<0.001$} & \multicolumn{2}{|l|}{$P=0.017$} & \multicolumn{2}{|l|}{$P<0.001$} & \multicolumn{2}{|l|}{$P=0.013$} \\
\hline
\end{tabular}

dicate that the polyp and adenoma detection levels may be improved with NER1006 compared with OSS or 2 L PEG.

Our results specifically suggest that there is a double chance to detect at least three adenomas in $\mathrm{OO}$ male patients when using NER1006 versus OSS or 2 L PEG. It is thus possible to improve the ADR or PDR in each male patient, ensuring accurate detection and safe removal of the adenomas/polyps. With the proper detection of adenomas/polyps, the clinical guideline re- commendations on the returning of patients with less than five adenomas per patient to screening will apply [6].

NER1006 may also present an increased patient benefit, particularly compared with OSS, by permitting both breakfast and lunch. Avoidance of unnecessary fasting periods may improve the nutritional status of elderly and hospitalized patients [22]. NER1006 has also been found to be safe and tolerable $[18,19$, 23]. 
- Table 6 Use of multiple logistic regression to predict the detection of male patients with three or more adenomas.

\begin{tabular}{|c|c|c|c|c|}
\hline & Odds ratio & $\mathrm{Cl} 2.5 \% \mathrm{LCL}$ & Cl $97.5 \%$ UCL & $P$ value \\
\hline \multicolumn{5}{|l|}{ All males, $n=514$} \\
\hline - Intercept & 0.015 & 0.002 & 0.101 & $<0.001$ \\
\hline - MORA trial & 0.274 & 0.134 & 0.553 & $<0.001$ \\
\hline - Age & 1.049 & 1.018 & 1.082 & 0.002 \\
\hline - NER1006 & 1.902 & 1.045 & 3.526 & 0.037 \\
\hline - IV fluids received during colonoscopy & 0.211 & 0.097 & 0.443 & $<0.001$ \\
\hline \multicolumn{5}{|l|}{ OO males, $n=419$} \\
\hline - Intercept & 0.010 & 0.001 & 0.083 & $<0.001$ \\
\hline - MORA trial & 0.274 & 0.126 & 0.582 & $<0.001$ \\
\hline - Age & 1.056 & 1.022 & 1.095 & 0.002 \\
\hline - NER1006 & 2.049 & 1.082 & 3.973 & 0.030 \\
\hline - IV fluids received during colonoscopy & 0.233 & 0.105 & 0.507 & $<0.001$ \\
\hline \multicolumn{5}{|c|}{$\begin{array}{l}\text { Of the } 20 \text { variables (with discrete values inside the parenthesis), the ones that entered into the backward elimination method to optimize the predictive model in- } \\
\text { cluded: MORA trial }(1 / 0) \text {, Age (years), BMI }(\mathrm{kg} / \mathrm{m} 2) \text {, the reason for colonoscopy: screening }(1 / 0) \text {, surveillance }(1 / 0) \text {, or diagnostic }(1 / 0) \text {, bowel preparation with } 1 \mathrm{~L} \\
\text { NER } 1006(1 / 0) \text {. Included variables capturing the patient medical history were diabetes }(1 / 0) \text {, renal insufficiency }(1 / 2 / 3 \text { for none/mild/moderate), cardiac condition } \\
(1 / 0) \text {, diverticular disease }(1 / 0) \text {, ongoing IBD }(1 / 0) \text {. Variables for medical treatments other than bowel preparation were gastrointestinal mobility inhibitors }(1 / 0) \text {, } \\
\text { gastrointestinal motility stimulants }(1 / 0) \text {, general anesthetics }(1 / 0) \text {, analgesics }(1 / 0) \text {, sedatives }(1 / 0) \text {, IV fluids }(1 / 0) \text {. Finally, the time-lapse from the end of prep to } \\
\text { the start of colonoscopy }(1 / 0 \text { for }<6 \text { hours } / 6 \text { + hours) and ADR } 3 \text { were the outcome variables. } \\
\text { IV, intravenous; IBD, inflammatory bowel disease; BMI, body mass index; ADR, adenoma detection rate. }\end{array}$} \\
\hline
\end{tabular}

This study has several strengths. We used relatively simple and established analyses to demonstrate clear and consistent results. The underlying data came from two highly similar prospectively randomized and treatment-blinded phase 3 clinical trials comparing NER1006 with two widely used bowel preparations, which are both known for their high efficacy, but which, between them, also have a comparable cleansing efficacy. While the randomization for the presence of adenoma was not possible, the real-world relevance in this analysis was compelling, given that both the superior cleansing assessment and the improved lesion detection were based on evaluations by the site endoscopists.

Our study has some limitations too. First, the post hoc analysis is not a prospectively randomized clinical trial. Second, we did not determine the size or morphology of detected polyps and adenomas. It would have been valuable to know the ratios between large or small and diminutive adenomas and between flat sessile serrated polyps and other polyps. Although we used ADR, which is a primary colonoscopy quality indicator used in all patients [24], the success of adenoma detection is reliant on the experience of the site endoscopists.

\section{Conclusions}

This exploratory analysis shows that $1 \mathrm{~L}$ NER1006 enables the detection of more males with ADR3 + and ADR5 + for frequent surveillance than comparator solutions. Future studies in the form of larger prospective clinical trials are warranted to verify the hypothesis that NER1006 can reproducibly allow detection of more adenomas in high-risk patients versus current alternatives.

\section{Acknowledgements}

The authors thank all the investigators, trial personnel, and participating patients for their contributions to the two underlying clinical trials that enabled our post hoc analysis. The authors would like to thank Norgine Ltd for making the trial data available for this post hoc analysis. We also specifically thank the authors of the primary trial publications for their pivotal scientific contributions: The MORA trial publication authors Lucy B. Clayton and Richard $\mathrm{Ng}$ Kwet Shing and the NOCT trial publication authors Michael P. DeMicco, Lucy B. Clayton, and Jeff Pilot. Medical writing assistance was provided by Azhaar Ashraf, funded by Norgine. The authors would finally like to thank Lucy Clayton, Richard Ng Kwet Shing, and Hosnieh Fathi at Norgine for their valuable comments during the preparation of this manuscript.

\section{Competing interests}

Dr. Epstein was an investigator in the NOCT study and has acted as a safety advisor for Aspire Bariatrics, a consultant for Zx Pharma and IM HealthScience, and a speaker for Daichi Sankyo and Pfizer. Dr. Halonen is an employee of Norgine. Dr. Sharma has served as a consultant for Boston Scientific and received grants from CDx Labs, US Endoscopy, and Medtronic. 
Clinical trial

Clinical.Trials.gov

NCT02254486

Clinical.Trials.gov

NCT02273167

TRIAL REGISTRATION: Both analyzed trials NOCT (NCT02254486) and MORA (NCT02273167) have been registrated as Multicenter Randomized Parallel Group Phase III Studies. Further details are available on clinicaltrials.gov

\section{References}

[1] Corley DA, Jensen CD, Marks AR et al. Adenoma detection rate and risk of colorectal cancer and death. N Engl J Med 2014; 370: 12981306

[2] Kaminski MF, Wieszczy P, Rupinski M et al. Increased rate of adenoma detection associates with reduced risk of colorectal cancer and death. Gastroenterology 2017; 153: 98-105

[3] Hartstein JD, Vemulapalli KC, Rex DK. The predictive value of small versus diminutive adenomas for subsequent advanced neoplasia. Gastrointest Endosc 2020; 91: 614-621.e6

[4] Kim NH, Jung YS, Park JH et al. Risk of developing metachronous advanced colorectal neoplasia after resection of low-risk diminutive versus small adenomas. Gastrointest Endosc 2020; 91: 622-630

[5] Gupta S, Lieberman D, Anderson JC et al. Recommendations for follow-up after colonoscopy and polypectomy: a consensus update by the US multi-society task force on colorectal cancer. Gastroenterology 2020; 158: 1131-1153.e5

[6] Hassan C, Antonelli G, Dumonceau JM et al. Post-polypectomy colonoscopy surveillance: European Society of Gastrointestinal Endoscopy (ESGE) Guideline - Update 2020. Endoscopy 2020; 52: 687-700

[7] Hassan C, Manning J, Álvarez González MA et al. Improved detection of colorectal adenomas by high-quality colon cleansing. Endosc Int Open 2020; 8: E928-E937

[8] Mauri G, Sartore-Bianchi A, Russo AG et al. Early-onset colorectal cancer in young individuals. Mol Oncol 2019; 13: 109-131

[9] Siegel RL, Torre LA, Soerjomataram I et al. Global patterns and trends in colorectal cancer incidence in young adults. Gut 2019; 68: 21792185

[10] Vuik FE, Nieuwenburg SA, Bardou M et al. Increasing incidence of colorectal cancer in young adults in Europe over the last 25 years. Gut 2019; 68: 1820-1826
[11] Bailey CE, Hu CY, You YN et al. Increasing disparities in the age-related incidences of colon and rectal cancers in the United States, 19752010. JAMA Surg 2015; 150: 17-22

[12] Waldmann E, Heinze G, Ferlitsch A et al. Risk factors cannot explain the higher prevalence rates of precancerous colorectal lesions in men. Br J Cancer 2016; 115: 1421-1429

[13] Rawla P, Sunkara T, Barsouk A. Epidemiology of colorectal cancer: incidence, mortality, survival, and risk factors. Prz Gastroenterol 2019; 14: 89-103

[14] IQVIA MIDAS 2020: OneView and the IQVIA World Review Pack; PLENVU procedures worldwide since launch. Data collected until June 2020 (August 2020 in some countries).

[15] Repici A, Coron E, Sharma P et al. Improved high-quality colon cleansing with $1 \mathrm{~L}$ NER1006 versus $2 \mathrm{~L}$ polyethylene glycol + ascorbate or oral sulfate solution. Dig Liver Dis 2019; 51: 1671-1677

[16] Epstein MS, Benamouzig R, Halonen J et al. High-quality colon cleansing and multiple neoplasia detection with $1 \mathrm{~L}$ NER 1006 versus mid-volume options: post hoc analysis of phase 3 clinical trials. Endosc Int Open 2020; 8: E628-E635

[17] Maida M, Sinagra E, Morreale GC et al. Effectiveness of very low-volume preparation for colonoscopy: a prospective, multicenter observational study. World J Gastroenterol 2020; 26: 1950-1961

[18] Bisschops R, Manning J, Clayton LB et al. Colon cleansing efficacy and safety with $1 \mathrm{~L}$ NER 1006 versus $2 \mathrm{~L}$ polyethylene glycol + ascorbate: a randomized phase 3 trial. Endoscopy 2019; 51: 60-72

[19] DeMicco MP, Clayton LB, Pilot J et al. Novel 1 L polyethylene glycolbased bowel preparation NER1006 for overall and right-sided colon cleansing: a randomized controlled phase 3 trial versus trisulfate. Gastrointest Endosc 2018; 87: 677-687.e3

[20] Gu P, Lew D, Oh S] et al. Comparing the real-world effectiveness of competing colonoscopy preparations: results of a prospective trial. Am J Gastroenterol 2019; 114: 305-314

[21] DerSimonian R, Laird N. Meta-analysis in clinical trials. Control Clin Trials 1986; 7: 177-188

[22] Nunes G, Barata AT, Santos CA et al. Nutritional deficiency during colonoscopy preparation: the forgotten iatrogeny. Rev Esp Enferm Dig 2018; 110: 285-291

[23] Repici A, Hassan C, Hoorn EJ et al. NER1006 is clinically safe. Gastrointest Endosc 2019; 89: 908-909

[24] Anderson JC, Butterfly LF. Colonoscopy: quality indicators. Clin Transl Gastroenterol 2015; 6: e77 